\title{
ORIGINAL ARTICLE PRECLINICAL EVALUATION OF THE VASCULAR EFFECTS OF REJUVENATE® COBALT CHROMIUM CORONARY STENT SYSTEM IMPLANTED IN THE PORCINE CORONARY IN STENT RESTENOSIS MODEL
}

\author{
Murtaza Najabat Ali ${ }^{1}$, Mariam Mir' ${ }^{\text {, Piotr Buszman }}{ }^{2}$ \\ ${ }^{1} \mathrm{~N}$-ovatove Health technologies, National University of Sciences \& Technology (NUST), Islamabad, Pakistan, ${ }^{2}$ Center for Cardiovascular \\ Research and Development, American Heart of Poland
}

\begin{abstract}
Objectives: The main objective of the study was to evaluate the implantation, safety, and vascular tissue effects of REJUVENATE® Cobalt Chromium Bare Metal Stent in the Porcine Coronary Restenosis model at short, mid and long-term follow up.

Methodology: A total of 18 REJUVENATE $®$ bare metal stents $(\mathrm{CoCr}, 95 \mu \mathrm{m}$ strut thickness) were implanted into the coronary arteries (RCA, LCX and LAD) of the six pigs. Throughout the study period, stent patency, vascular response, percent stenosis, late lumen loss and performance of stent were evaluated by angiography, optical coherence tomography (OCT), and Histopathology (includes both Histopathology and histomorphometry) at 30, 90, and 180 days and compared with the results of control Bare Metal Stent Coflexus ${ }^{\circledR}$. The mean, variance and standard deviation was analyzed using SPSS software.

Results: OCT and QCA analysis demonstrated two cases of binary restenosis at 30 days followup and two at 90 days follow-up (33\%). The mean area stenosed for Rejuvenate ${ }^{\circledR}$ was $34 \%$ as compared to $24 \%$ of Coflexus $®$ and at 180 days follow up, it decreased to $21 \%$. The histopathology analysis demonstrated favorable safety with acceptable levels of neointimal formation, with decreased levels of inflammation and complete endothelialization in 180 days . Conclusion: This study proved the potential safety of REJUVENATE $®$ in porcine model and supports that this stent can move forward for the human trials.

Keywords: Drug Eluting Stents, Animal Studies, Porcine Model, Restenosis, Angioplasty, Dual antiplatelet therapy

Citation: Ali MN, Mir M, Buszman P. Preclinical Evaluation of the Vascular Effects of Rejuvenate® Cobalt Chromium Coronary Stent System Implanted in the Porcine Coronary in Stent Restenosis Model. Pak Heart J. 2021;54(02):132-138. DOI: https://doi.org/10.47144/phj.v54i2.2087
\end{abstract}

\section{INTRODUCTION}

According to WHO (World Health Organization), heart diseases are leading cause of death in United States ${ }^{1}$ as it causes death of one person in every 37 seconds. ${ }^{2}$ According to a report published in 2017, Coronary Heart Disease was the major cause of death resulting in $42.3 \%$ deaths in United States, followed by stroke (17.0\% deaths), High Blood Pressure (10.5\% deaths), Heart Failure (9.4\% deaths), diseases of the arteries (2.9\% deaths), and other cardiovascular diseases ( $17.6 \%$ deaths). ${ }^{3}$

Coronary heart disease is attributed as complete blockage or narrowing of the coronary arteries that carry blood and oxygen to the heart. ${ }^{4}$ The reason behind this situation is atherosclerosis which is a buildup of fatty material and plaque inside the coronary arteries. This plaque can block the flow of blood towards muscles and other body parts which causes the severe chest pain and heart attack. To reopen the coronary arteries Percutaneous Transluminal Coronary Angioplasty (PTCA) is performed which is a minimally invasive procedure; however, reappearance of plaque or restenosis follows PTCA in $30-40 \%$ of coronary lesions within 6 months. ${ }^{5}$

To solve the problems of PTCA, mesh like metallic hollow tubes were introduced which are known as Bare-metal coronary artery stents (BMS). BMS is used in percutaneous coronary intervention (PCI) for a variety of indications, including stable and unstable angina, acute myocardial infarction (MI), and multiple-vessel disease.

BMS has been introduced in the market since decades ago and there are large number of BMS with varying properties such as metal platform, strut thickness or cell design. REJUVENATE® is manufactured in Pakistan for the native population. This paper proves the potential safety of REJUVENATE® through animal studies using porcine coronary stent restenosis model. It indicates the possible safety of REJUVENATE® which can be further validate through different studies.

Scientists have been using porcine models for decades to analyze the safety of cardiac stents before their implantation inside humans. The 
similarities between cardiovascular system of porcine and humans makes it best choice to analyze the effects of any implants. The distribution of epicardial coronary artery of porcine resembles with humans. The bifurcation of left main coronary artery of porcine into left anterior descending (LAD) and circumflex $(\mathrm{Cx})$ coronary arteries is similar to humans and their diameters ( 2 to $4 \mathrm{~mm}$ ) also closely resembles to humans. Despite of some differences, the basic cardiac hemodynamic parameters and platelet characteristics are similar between both species. ${ }^{6}$ In addition, the angioplasty procedure for porcine is also similar to human. Cardiologists can use same apparatus to treat both humans and porcine. ${ }^{7}$

The procedure of PCI is same for porcine and humans. Like human PCI, the animals are anesthetized, and the vascular sheath is inserted inside blood vessel of animal. Fluoroscopic guidance is used for the advancement of catheter in vessels. The coronary angiograms are recorded to identify the suitable segments for stent implantation. Generally, Quantitative angiography (QCA) is performed to measure the vessel diameters. Optical coherence tomography (OCT) is modern and preferred method to obtain high resolution images and accurate information. Unlike humans, in animals, 2 or more sites are implanted with stents. ${ }^{8}$

The main objective of this study was to analyze the deliverability and safety by examining the vascular response of animals. Prior to the experiment atherosclerosis was induced in these animals. The effect of stent placement on vascular remodeling was evaluated by comparing the vessel morphology of the stented coronary segments with the stented segments of commercially available Coflexus Stent. The optical coherence tomography and quantitative coronary angiography was carried out for the quantitative measurements and histopathology was performed to observe the vascular response. The experiments were performed on porcine coronary model which is accepted as a standard model for CAD studies.

\section{METHODOLOGY}

The current pre-clinical study was conducted in 2019 at Pre-clinical Research Laboratory, Center of Cardiovascular Research and Development, American Heart Poland. The duration of this trial was 6 months, and the animals of choice were Domestic Pigs (Sus scrofa domesticus). The porcine model was selected for this study due to the anatomical and physiological similarities with the human coronary arteries. ${ }^{9}$ Scientists have been using Porcine model for testing intracoronary devices since decades. The results were compared with Coflexus Stent [Balton, Poland] which is a 70 $\mu \mathrm{m}$ thick cobalt chromium bare metal stent. This stent is famous for its high flexibility and ultra-low crossing profile. Approval from Ethical Committee of American Heart Poland was obtained before starting the trial (Ethics committee approval certificate number is 83118).

The Test article was REJUVENATE $®$ cobalt chromium bare-metal stent for implantation in coronary arteries and treatment of coronary artery disease by interventional technique. REJUVENATE® comprises of the following components:

- A balloon expandable MP35NLT Cobalt chromium coronary stent

- A rapid exchange delivery PTCA balloon dilation catheter

The stent was pre-mounted crimped on the balloon of a PTCA catheter for endovascular delivery and implantation at the desired site. The delivery system consisted of a rapid-exchange PTCA catheter with a balloon. Nominal pressure of the balloon (for the test article) was 11 ATM and its rated burst pressure was 15 ATM. The stent sizes used in this study were $3.00 \times 20 \mathrm{~mm}$. The stent system was compatible with $5 \mathrm{~F}$ guiding catheter and 0.014 " guide wire and the usable catheter length is $135+/-5 \mathrm{~cm}$.

Coflexus Bare Metal Stent [Balton, Poland] was implanted as control stent. The Cobalt Chromium metal platform of this stent is $70 \mu \mathrm{m}$ thick and is famous for its flexibility. Unlike REJUVENATE®, Coflexus has closed cell design. Its cell area is also wider as it was specially designed for branched coronary arteries.

Total eight Domestic Pigs were acquired from Institute of Animal Production Grodziec Śląski, Poland. The herd was free from common domestic swine diseases and vaccinated accordingly to standard operating procedures. Animals were monitored for the signs of disease during the acclimation period before enrolling them into the study. At the time of study, the pigs were 3 months old, Castrated male/Female and had 35 to $40 \mathrm{~kg}$ weight.

For the vascular injury, the test animals were subjected to overstretch balloon injury. The animals were sedated, and injury was performed with an angioplasty balloon larger in size than the reference vessel diameter. The balloon was inflated. After the completion of the injury, the angioplasty balloon was withdrawn. ${ }^{10}$

Second angioplasty was performed to insert the stent inside the vessel. Six animals were implanted with REJUVENATE® and two with Coflexus. Each animal was implanted with one stent in each one of 
the coronary arteries (a) left circumflex artery (LCX), (b) left anterior descending coronary artery (LAD), and (c) right coronary artery (RCA). All the animals received $100 \mathrm{mg}$ of aspirin and $75 \mathrm{mg}$ of clopidogrel daily until death. After 30, 90 and 180 days the animals were evaluated by angiography, optical coherence tomography (OCT), and Histopathology (includes both Histopathology and histomorphometry).

Clinical follow up was carried out at 30 days, 90 days and 180 days after the stent implantation. Restenosis was defined as decrease in vessel diameter after stent implantation and it was calculated by measuring the diameter and area of stenosed region. Procedural success was defined as no incidence of revascularization, vessel failure and cardiac death. The lumen area gain was defined as the difference in lumen area before stent implantation and after stent implantation. Late lumen loss is defined as the difference between the diameter of a stented segment post-procedure compared with the follow-up angiogram at six or nine months.

Quantitative Coronary Angiography (QCA) carried out for the measurements of the vessel at the target site for selecting appropriate size for stent implantation. At the designated endpoint (30, 90, and 180 days), animals were euthanized under general anesthesia by IV injection of a commercially available euthanasia solution.

Optical coherence tomography (OCT) is an invasive imaging technique that produces high resolution intracoronary images. OCT was performed only at follow up time-points. The purpose of OCT was to validate the results of QCA and get a pictorial description of area of interest.

For histopathological analysis, samples were obtained from the arterial segments containing stents. The segments were cut into slices using Leica microtome and stained with resins for histomorphometric analysis. After staining, the lumen cross-sectional area, stent cross-sectional area and Neointimal area were assessed. The percentage of vascular lumen narrowing (\% area stenosis) was calculated according to the following formula: ${ }^{11}$

$\%$ Stenosis $=($ IEL area - lumen cross-sectional area)/IEL area $x 100$

For the measurement of each parameter, proximal, medial and distal part of the stent were measurement and subsequently, a mean value was calculated. The qualitative analysis to assess arterial wall integrity and the presence of endothelium, fibrin, thrombi or focal necrosis was carried out.
Each sample was evaluated for inflammation and neointimal score. Inflammatory response was evaluated using a four-grade scale (grades from 0 to 3 ) described by Kornowski et al. Inflammation score on a scale of 0 to 4 , Neointima maturity was observed and qualitatively analyzed.

After obtaining the results, data was analyzed through statistical tool SPSS both qualitatively and quantitatively. Results are expressed as mean \pm standard deviation and percentages. The results were compared with the results of control stent. Angiographic and histomorphometric data were analyzed using SPSS software (19.0 system).

\section{RESULTS}

In the current study, six animals were implanted with stents. Each animal three stents, one for each coronary artery (LAD, LCX and RCA). During the whole process, animals were alert and responsive without any evidence of abnormal temperature or weight loss or other health complications. The blood flow remained within the normal limits at all time points evaluated. The overall combined mortality rate of the study was less than $1 \%$.

Quantitative coronary angiograms recorded at 30, 90 and 180 days follow-up and were analyzed at the laboratory. Baseline diameters and stenosed area were measured. The results demonstrated that baseline vessel diameters were similar between 30 , 90, and 180-days follow-up $(2.2 \mathrm{~mm}, 2.3 \mathrm{~mm}$ and $2.2 \mathrm{~mm}$ respectively). Similarly, the overstretch expressed as proportion between baseline and post implantation was $110 \%$. There were two cases of binary restenosis at 30-day follow-up and two at 90day follow-up (33\%).

Late lumen loss is the difference between the diameter of a stented segment post-procedure compared with the follow-up angiogram at six or nine months. REJUVENATE $₫ \square$ was studied for late lumen loss at 30 days and 90 days. Through QCA and OCT the lumen loss was measured, and the results are shown in Figure 1 for 30 and 90 days follow up. Late lumen loss was measured by measuring the difference in the post stent implantation vessel diameter and vessel diameter after a 30- and 90-days post implantation.

Area stenosis was measured only on cross sectional images. Figure 1 demonstrates percentage area stenosed which was calculated by measuring the minimum diameter of coronary vessel after stent implantation and 30 and 90 days follow up. The results suggest $66 \%$ of area stenosis which was decreased to $55 \%$ at 90 days follow up. QCA was not carried out for Coflexus stent and it only demonstrated the results within the test group. 


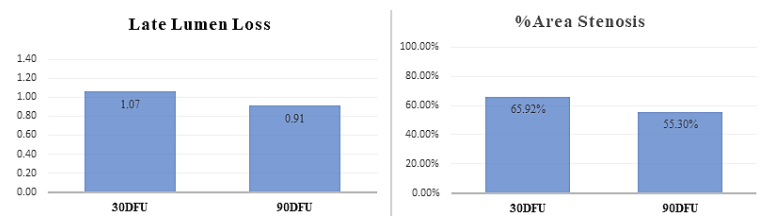

Figure 1: comparison of 30 days and 90 days follow up (a) Late Lumen Loss (b) Area stenose

OCT was used to compare the lumen diameter, area stenosis and diameter stenosis of REJUVENATE® with commercially available Coflexus Stent. The analysis was done at 30 days, 90 days and 180 days. The images of each follow up are given in Figure 2. Through this analysis, diameter of stenosed area and late lumen was calculated and the values were compared to the QCA results.

Results of 30 days follow up demonstrated that all stent diameters were comparable between the test group, however the Coflexus stent has numerically larger diameter. Values for REJUVENATE® Cobalt Chromium Coronary System were numerically higher for neointimal hyperplasia parameters when matched against Coflexus®. Nonetheless, p values showed no statistical difference for \% AS, \%DS, and neointima area (NA). P values for area stenosed are 0.18 and for diameter stenosed are 0.21 .

The results of 90 days follow up exhibited the \% area stenosis and \% diameter stenosis were greater at $90 \mathrm{DFU}$ than for the subjects in the $30 \mathrm{DFU}$ group. In one pig, OCT imaging confirmed abnormalities in this case and its reason could be late stent malposition or unexpanded stent at implantation. Due to the lack of baseline OCT images, it is not possible to establish whether that pig had under-expanded stents at implantation or due to late stent malposition.

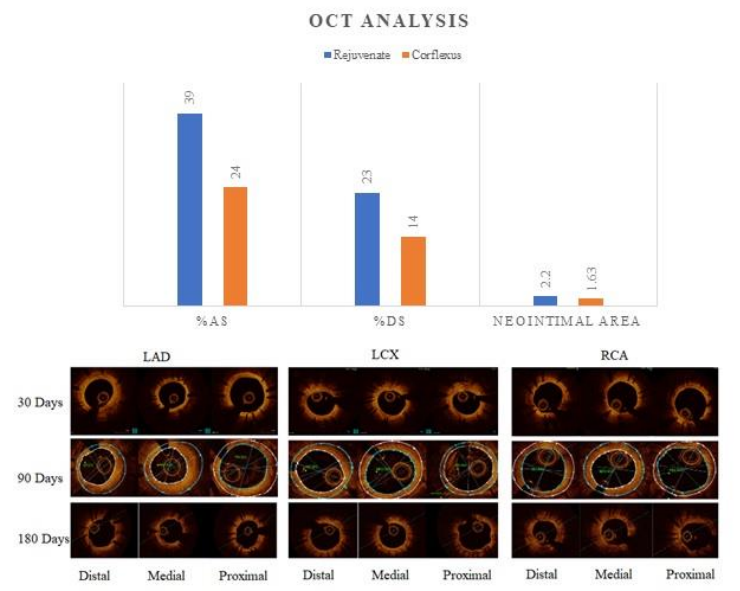

Figure 2: OCT analysis (a) Performance at 30 days (b) OCT images at 30, 90 and 180 days
At 180 days follow up, \% area stenosis and \% diameter stenosis were lower at 180 DFU than for the subjects in the 90 DFU group.

Histomorphometry measures the shape or form of a tissue. In this study, lumen area, stent area, neointimal area and area stenosis are measured at 30, 90 and 180 days through Histomorphometry which are given in Figure 3. The average area stenosis was $51 \%$, and the binary restenosis rate was $22 \%$ (4/18). Results demonstrated that compared to QCA, area stenosis was slightly lower, with a maximal area stenosis of $93 \%$. Mean neointima area was $3.63 \mathrm{~mm}^{2}$. The mean neointima thickness was calculated from lumen area and IEL area was found to be around $0.53 \mathrm{~mm}$.

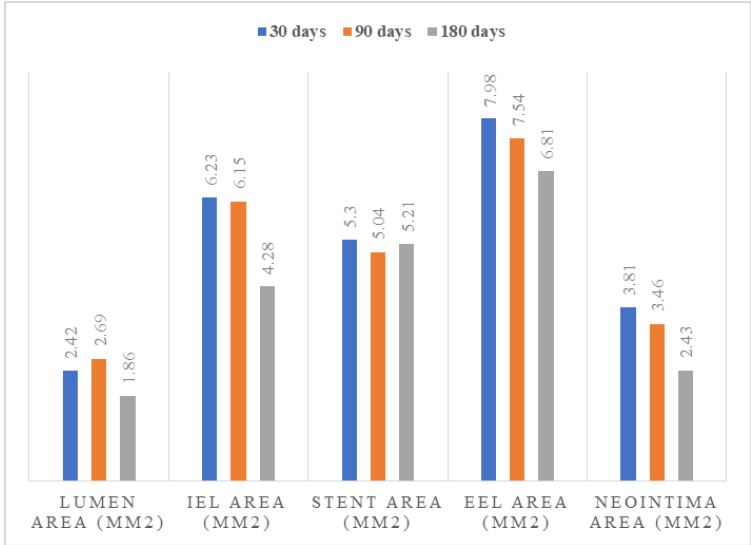

Figure 3: Histomorphometric analysis of explanted stents at 30, 90 and 180 DFU

The procedures were completed without any complications, and all swine survived until the end of the follow-up period. Histopathology was not performed for control group animals. At 30 days follow up, there was mild inflammation in LAD and LCX and moderate inflammation in RCA. At 90 days follow up, only LAD demonstrated moderate inflammation and at 180 days, all the vessels exhibited mild or no inflammation.

The results of 30 days follow up of RCA, LCX and LAD demonstrated that neointima was immature and composed of myofibroblasts over $25 \%$ and extracellular matrix. In RCA, Internal elastic lamina was retained at first three levels, whereas at level 4th and 5th were disrupted. Endothelial lining was present at nearly half of the lumen. In LCX, Internal elastic lamina was disrupted in first 4 levels, but in last levels was only compressed. In LAD, Internal elastic lamina was disrupted in 1st and last parts.

In 90 days, follow-up, neointima was immature at all levels and was composed of myofibroblasts over $25 \%$ and extracellular matrix. In RCA, internal elastic lamina was disrupted in all levels, whereas external elastic was disrupted only at level 6th. 
Endothelial coverage was focal at all levels. In LCX, Internal elastic lamina was disrupted in all levels, whereas external elastic was disrupted at levels 2-4. Endothelial coverage was focal at all levels. In LAD, Internal elastic lamina was disrupted in all levels. Endothelial coverage was focal at all levels.

At 180 days, Neointima consisted of numerous myofibroblasts and fibroblasts, exceeding $50 \%$ of cells, extracellular space was reduced. Thus, neointimal covering was compact.

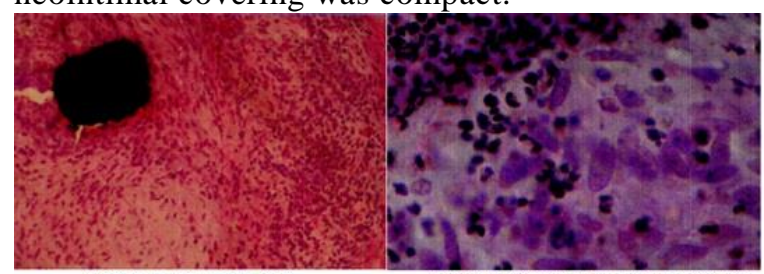

(A) Moderate Inflammation (RCA)

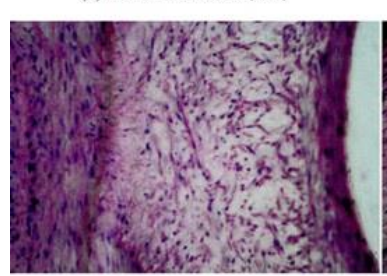

(C) Neointima with predominant extracellular matrix

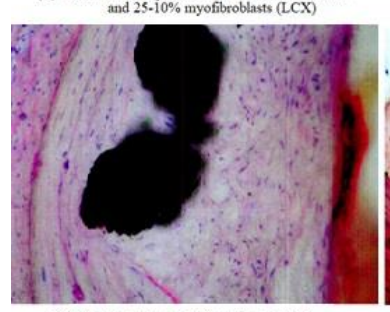

(E) No Inflammatory Infilterations in RCA

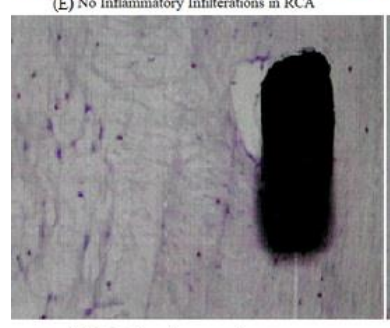

(G) No Inflummation around struts
(B) Numerous Nuetrophil and a few cosinophil inside

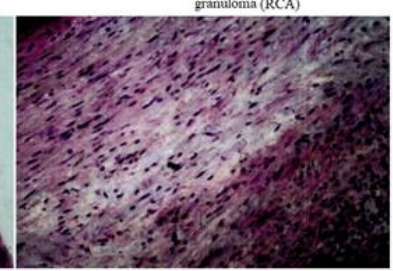

(D) Compact myofibrillar neointima (LAD)

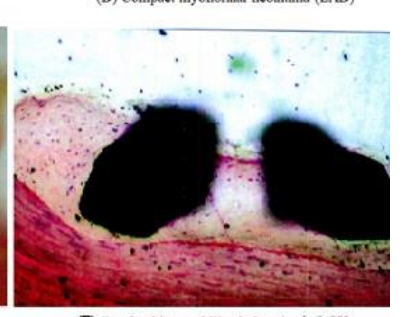

(F) Retained intemal Elastic Lamina in LCX

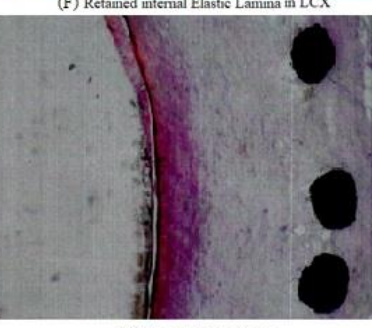

Figure 4: Histopathology results $(A, B, C, D) 30$ days, (E, F) 90 days $(G, H) 180$ days follow up

In RCA, Internal elastic lamina was evidently disrupted at all levels, but without laceration of media. Linear endothelial lining was best seen at distal level. It should be emphasized, that in all levels endothelial cells covered nearly $50 \%$ of lumen. Neointima showed more advanced maturity at proximal and medial level, whereas distant level was less compact poorer in myofibroblasts. In LCX, Internal elastic lamina was evidently disrupted at medial and distal levels, but without laceration of media. Linear endothelial lining was present focally, not exceeding $50 \%$ of lumen. In LAD, Internal elastic lamina was disrupted at proximal level, at distant level focally external elastic membrane was disrupted. The focal endothelial lining was seen at proximal level (nearly $50 \%$ of lumen), whereas at distant level diminished to focal one quarter. Neointimal image at all levels showed nearly $50 \%$ or more myofibroblasts.

\section{DISCUSSION}

The introduction of intravascular stents to clinical practice marked the beginning of a new era in the history of percutaneous coronary intervention. Stents reduced the restenosis rate and increased the safety and efficacy of procedures. $^{12}$ However, it soon became clear that traditional stainless-steel stents could not cope with certain difficult clinical situations and that the development of a new stent type was needed. ${ }^{13}$ The new metal cobalt-chromium was introduced to improve the performance of DES by augmenting mechanical properties and thinner struts. REJUVENATE® is a cobalt-chromium stent with $95 \mu \mathrm{m}$ strut thickness. In this study, the potential safety of REJUVENATE® at 30, 90, and 180 days in animals and the results of this study will straighten the path for human studies.

The domestic porcine model was chosen in our study because it is considered as a standard for stent testing. In many studies, several parameters have been studied which included degree and uniformity of neointimal coverage, presence of uncovered struts, thrombus formation, arterial healing, restenosis rate, external elastic lamina (EEL) area, internal elastic lamina (IEL) area, lumen area, neointimal area, medial area, lumen equivalent diameter, IEL equivalent diameters and percent area stenosis. ${ }^{14-16}$

Neointimal hyperplasia is a post stent implantation response which is associated with vascular remodeling of coronary vessels. The incidence of remodeling of vessels is carried out by migration and proliferation of smooth muscle cells of vessels into tunica intima. If remodeling of vessels is not balanced, it will lead to undesirable vessel wall thickening resulting in loss of luminal patency. ${ }^{17}$ REJUVENATE® demonstrated $3.8 \mathrm{~mm} 2,3.4$ $\mathrm{mm} 2$ and $2.4 \mathrm{~mm} 2$ neointimal area after 30, 60 and 90 days respectively. Similar study was conducted on Multilink Vision BMS [Abbott Laboratories, USA] and Pro-Kinetic BMS [Biotronik, Germany] for 28 days and results demonstrated 1.29 and $1.63 \mathrm{~mm} 2$ neointimal area. $^{18}$ The neointimal area for REJUVENATE® was higher at 30 days study, however it decreased within 180 days which proved efficiency of the stent.

The relationship between the arterial injury, inflammation, neointimal thickness and lumen stenosis has already been established in various studies. ${ }^{19}$ The 
results demonstrated that at all levels, the inflammatory infiltrations, even minimal, were absent. At 30 and 90 days follow up, a little inflammation was observed at a few levels; however, at 180 days follow up, no inflammation or minimal inflammation was observed. The neointima was immature till 90 days but at 180 days follow up it was matured. Studies have demonstrated mild inflammations in pre-clinical studies of different stents at 28 days. $^{18,20}$ The inflammation caused by REJUVENATE® disappeared at 180 days follow up.

At proximal and medial level, the maturity was advanced as compared to distal level. The morphometric results showed acceptable level of neointimal vascular response and optimal healing at cellular level. The OCT images demonstrated homogenous pattern and smooth intima formation. The rate of arterial injury was minimal as only one pig showed signs of injury at one vessel due to malposition of stent, otherwise, no injury was observed throughout the study. The lumen areas of coronary arteries are associated with vessel enlargement remodeling. The lumen area also significantly decreased in 180 days follow up as compared to 30 days follow up. The similar pattern has been observed in previous studies. $^{21}$

In this study, porcine model was used to prove the safety of REJUVENATE® BMS may not precisely predict the effects due to differences in human and porcine vasculature. It cannot reflect the exact biological response of humans. Another limitation of this study was the availability of control stents as only one control BMS was used for the comparison purpose. Third limitation was that the control stent could not be studied in the histopathology tests due to availability of smaller number of samples. The head-to-head comparison of both stents in histopathology can portray a better picture.

\section{CONCLUSION}

REJUVENATE® Cobalt Chromium Coronary stent is manufactured indigenously in Pakistan for native population. In the current study, REJUVENATE® demonstrated acceptable levels of percent of area stenosis and percent of diameter stenosis. The morphometric and histopathological results exhibited acceptable level of neointimal vascular response and complete lesion healing in 180 days. In all tests, REJUVENATE® demonstrated non-inferiority results as compared to Coflexus ${ }^{\circledR}$ stent and proved its potential safety for further studies.

In future, the REJUVENATE® will be studied on larger number of animals. It will also be studied against multiple control group of stents for the better comparison. Also, the human trials will be conducted to observe the safety of REJUVENATE® on humans.

\section{AUTHORS' CONTRIBUTION}

MNA: Concept and design, data acquisition, interpretation, drafting, final approval, and agree to be accountable for all aspects of the work. MM, PB: Data acquisition, interpretation, drafting, final approval and agree to be accountable for all aspects of the work.

Conflict of interest: Authors declared no conflict of interest.

\section{REFERENCES}

1. Rana JS, Khan SS, Lloyd-Jones DM, Sidney S. Changes in Mortality in Top 10 Causes of Death from 2011 to 2018. J Gen Intern Med. 2020;1-2. DOI: 10.1007/s11606-020-06070-z [Online ahead of print]

2. Prevention CfDCa. Underlying Cause of Death, 1999-2019. In: Prevention CfDCa, editor. CDC WONDER Online Database. Atlanta, GA: Centers for Disease Control and Prevention; 20182020. Available from: https://wonder.cdc.gov/ucdicd10.html

3. Benjamin EJ, Muntner P, Alonso A, Bittencourt MS, Callaway CW, Carson AP, et al. Heart Disease and Stroke Statistics-2019 Update: A Report From the American Heart Association. Circulation. 2019;139(10): e56-e528.

4. Barton M, Grüntzig J, Husmann M, Rösch JJFicm. Balloon angioplasty-the legacy of Andreas Grüntzig, MD (1939-1985). 2014;1:15.

5. Mani G, Feldman MD, Patel D, Agrawal CMJB. Coronary stents: a materials perspective. Biomaterials. 2007;28(9):1689-710.

6. Lowe H, Schwartz R, Neill B, Jang I-K, Hayase M, Rogers C, et al. The Porcine Coronary Model of In-Stent Restenosis: Current Status in the Era of Drug-Eluting Stents. Catheter Cardiovasc Interv. 2003;60:515-23.

7. Suzuki Y, Yeung AC, Ikeno F. The representative porcine model for human cardiovascular disease. J Biomed Biotechnol. 2011;2011:195483.

8. Iqbal J, Chamberlain J, Francis SE, Gunn J. Role of Animal Models in Coronary Stenting. Ann Biomed Eng. 2016;44(2):45365.

9. Hughes GC, Post MJ, Simons M, Annex BHJJoAP. Translational physiology: porcine models of human coronary artery disease: implications for preclinical trials of therapeutic angiogenesis. J Appl Physiol (1985). 2003;94(5):1689-701.

10. Suzuki Y, Lyons JK, Yeung AC, Ikeno FJJoIC. The porcine restenosis model using thermal balloon injury: comparison with the model by coronary stenting. J Invasive Cardiol. 2008;20(3):142.

11. Buszman P, Milewski K, Żurakowski A, Pająk J, Liszka $Ł$, Buszman P, et al. Novel biodegradable polymer-coated, paclitaxel-eluting stent inhibits neointimal formation in porcine coronary arteries. Kardiol Pol. 2010;68(5):509-15.

12. Serruys PW, De Jaegere P, Kiemeneij F, Macaya C, Rutsch W, Heyndrickx G, et al. A comparison of balloon-expandable-stent implantation with balloon angioplasty in patients with coronary artery disease. N Engl J Med. 1994;331(8):489-95.

13. Schmidt T, Abbott JD. Coronary Stents: History, Design, and Construction. J Clin Med. 2018;7(6):126.

14. Perkins LEL, Boeke-Purkis KH, Wang Q, Stringer SK, Coleman LA. XIENCE V V Everolimus-Eluting Coronary Stent System: A Preclinical Assessment. J Interv Cardiol. 2009;22(s1):S28-S40.

15. Sumida A, Gogas BD, Nagai H, Li J, King SB, 3rd, Chronos N, et al. A comparison of drug eluting stent biocompatibility between third generation NOBORI biolimus A9-eluting stent and second generation XIENCE V everolimus-eluting stent in a porcine coronary artery model. Cardiovascular revascularization medicine : including molecular interventions. Cardiovasc Revasc Med. 2015;16(6):351-7.

16. Takimura CK, Galon MZ, Gutierrez PS, Sojitra P, Vyas A, Doshi $\mathrm{M}$, et al. A new polymer-free drug-eluting stent with nanocarriers eluting sirolimus from stent-plus-balloon compared with bare- 
metal stent and with biolimus A9 eluting stent in porcine coronary arteries. Cardiovasc Diagn Ther. 2015;5(2):113-21.

17. Shoji M, Koba S, Kobayashi YJBri. Roles of bone-marrowderived cells and inflammatory cytokines in neointimal hyperplasia after vascular injury. Biomed Res Int. 2014;2014.

18. Wittchow E, Hartwig S. Still room for improvement: Preclinical and bench testing of a thin-strut cobalt-chromium bare-metal stent with passive coating. J Biomed Mater Res B Appl Biomater. 2017;105(6):1612-21.

19. Kornowski R, Hong MK, Tio FO, Bramwell O, Wu H, Leon MB In-Stent Restenosis: Contributions of Inflammatory Responses and Arterial Injury to Neointimal Hyperplasia. J Am Coll Cardiol. 1998;31(1):224-30

20. Sprague Eugene A, Tio F, Ahmed SH, Granada Juan F, Bailey Steven R. Impact of Parallel Micro-Engineered Stent Grooves on Endothelial Cell Migration, Proliferation, and Function. Circulation: Cardiovasc Interv. 2012;5(4):499-507.

21. Durand E, Sharkawi T, Leclerc G, Raveleau M, van der Leest M, Vert M, et al. Head-to-Head Comparison of a Drug-Free Early Programmed Dismantling Polylactic Acid Bioresorbable Scaffold and a Metallic Stent in the Porcine Coronary Artery. Circulation: Cardiovasc Interv. 2014;7(1):70-9.

\section{Address for Correspondence:}

Dr. Murtaza Najabat Ali, Department of Biomedical Engineering and Sciences (BMES), School of Mechanical and Manufacturing Engineering (SMME), National University of Sciences and Technology (NUST), Islamabad, Pakistan. Email: murtaza.bme@hotmail.com 